\title{
Molecular Layer-defined Transition of Carrier Distribution and Correlation with Transport in Organic Crystalline Semiconductors
}

Sai Jiang, Qijing Wang, Jun Qian, Jianhang Guo, Yiwei Duan, Hengyuan Wang, Yi Shi, ${ }^{*}$ and Yun Li ${ }^{*}$ National Laboratory of Solid-State Microstructures, School of Electronic Science and Engineering, Collaborative Innovation Center of Advanced Microstructures, Nanjing University, Nanjing, Jiangsu 210093, P. R. China.

\section{Corresponding Author}

*E-mail: yli@nju.edu.cn; yshi@nju.edu.cn. 


\section{Supplementary figures}

a

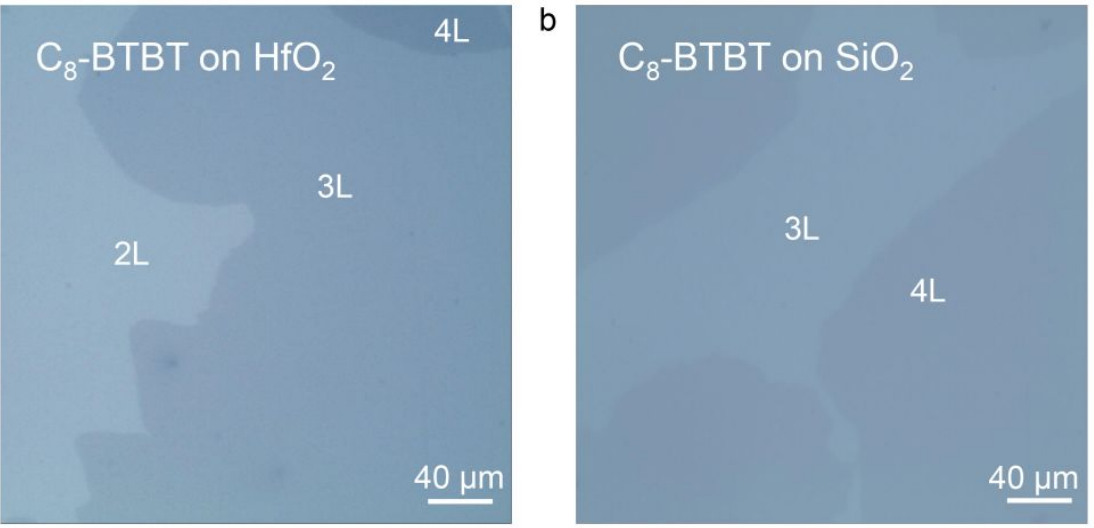

Figure S1. Optical microscopy characterization of $\mathrm{C}_{8}$-BTBT crystalline films. (a,b) Optical microscopy images of crystalline $\mathrm{C}_{8}$-BTBT with a step-and-terrace structure. The selected films exhibited large areas of up to hundreds of micrometres for $3 \mathrm{~L}$ and $4 \mathrm{~L} \mathrm{C}_{8}-\mathrm{BTBT}$ on the $\mathrm{HfO}_{2}$ and $\mathrm{SiO}_{2}$ substrates, respectively.

a
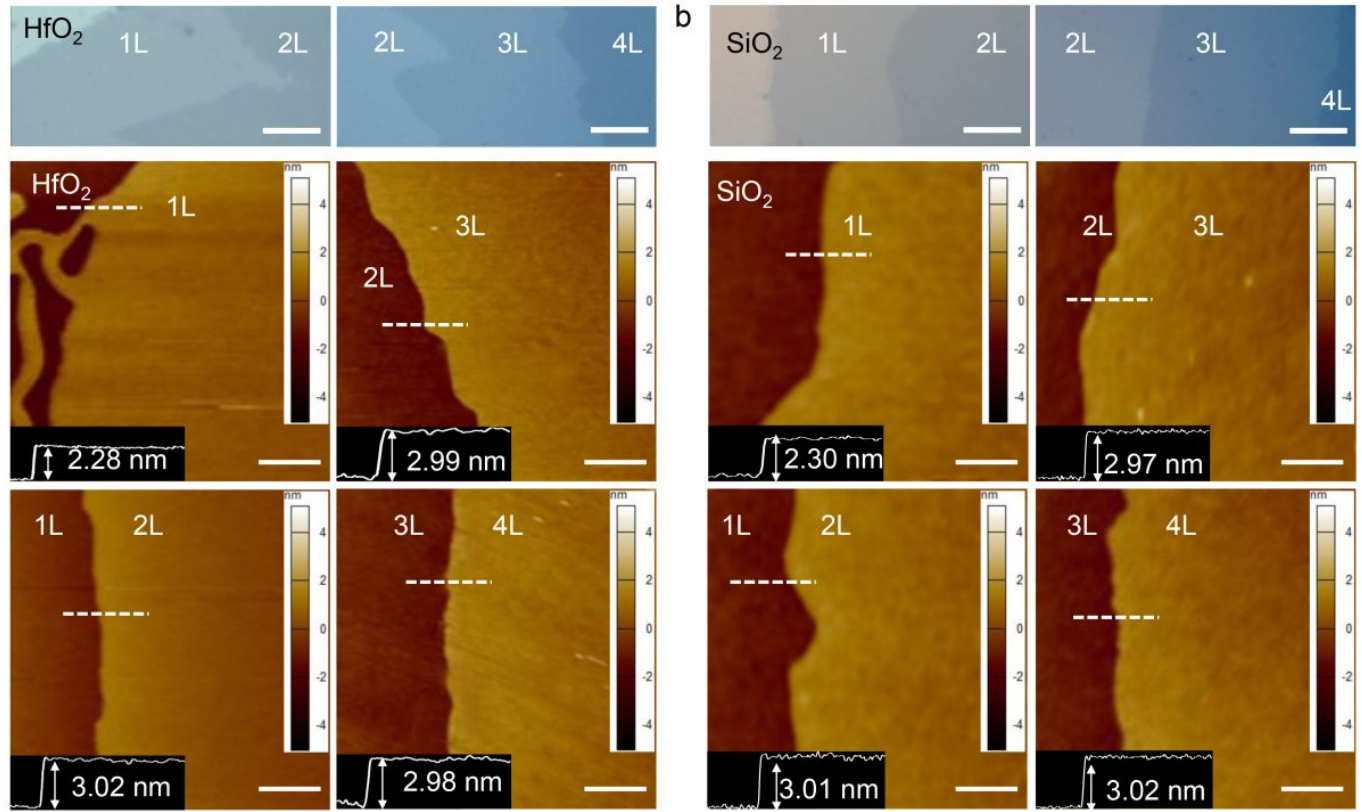

Figure S2. AFM characterization of $\mathrm{C}_{8^{-}}$BTBT crystalline films. (a,b) AFM morphology images of $\mathrm{C}_{8^{-}}$ BTBT films with one to four layers on the $\mathrm{HfO}_{2}$ (Left) and $\mathrm{SiO}_{2}$ (Right) dielectrics. The layer numbers are marked. The insets show the height profiles along the dashed lines. Scale bars of the optical microscopy images, $20 \mu \mathrm{m}$. Scale bars of the AFM morphology images, $4 \mu \mathrm{m}$ for $\mathrm{HfO}_{2}$ and $2 \mu \mathrm{m}$ for $\mathrm{SiO}_{2}$. 
Table S1. The lattice constant of $\mathrm{C}_{8}-\mathrm{BTBT}$ on $\mathrm{SiO}_{2}$ and $\mathrm{HfO}_{2}$ dielectric.

\begin{tabular}{ccccc}
\hline Dielectric & Layer Number & $a(\AA)$ & $b(\AA)$ & $\theta\left(^{\circ}\right)$ \\
\hline & $1 \mathrm{~L}$ & $6.60 \pm 0.13$ & $8.30 \pm 0.15$ & $89.5 \pm 1.4$ \\
$\mathrm{SiO}_{2}$ & $2 \mathrm{~L}$ & $6.36 \pm 0.10$ & $8.16 \pm 0.12$ & $88.4 \pm 1.2$ \\
& $3 \mathrm{~L}$ & $6.30 \pm 0.16$ & $8.13 \pm 0.14$ & $88.0 \pm 1.0$ \\
& $4 \mathrm{~L}$ & $6.29 \pm 0.12$ & $8.14 \pm 0.12$ & $87.9 \pm 1.4$ \\
& $1 \mathrm{~L}$ & $6.57 \pm 0.12$ & $8.28 \pm 0.13$ & $89.3 \pm 1.2$ \\
$\mathrm{HfO}_{2}$ & 2L & $6.32 \pm 0.11$ & $8.15 \pm 0.14$ & $88.2 \pm 1.1$ \\
& 3L & $6.28 \pm 0.12$ & $8.13 \pm 0.10$ & $88.0 \pm 1.0$ \\
& $4 \mathrm{~L}$ & $6.26 \pm 0.14$ & $8.12 \pm 0.09$ & $87.7 \pm 1.1$ \\
\hline
\end{tabular}




\section{Note 1. Extracting $C_{\mathrm{it}}$ and $\tau_{\mathrm{it}}$ by the AC conductance $G_{\mathrm{p}}$.}

The AC conductance $G_{\mathrm{p}}$ can be extracted from the measured capacitance $\left(C_{\mathrm{m}}\right)$ and conductance $\left(G_{\mathrm{m}}\right) .{ }^{1}$ Here, we neglect the bulk capacitance $C_{\text {bulk }}$ and the bulk resistance $R_{\text {bulk. }} G_{P}(\omega)=\mathrm{R} e$ $\left\{\left[\left(R_{m}+\left(i \omega C_{m}\right)^{-1}-\left(i \omega C_{o x}\right)^{-1}-\left(i \omega C_{\text {chain }}\right)^{-1}\right)\right]^{-1}\right\}$, and $R_{m}=\frac{G_{m}}{G_{m}^{2}+\omega^{2} C_{m}^{2}}$, where $\omega$ is the angular frequency, $C_{\mathrm{ox}}$ is the oxide capacitance, and $C_{\text {chain }}$ is the side-chain capacitance of $\mathrm{C}_{8}$-BTBT molecules.

The relation between $G_{\mathrm{p}}(\omega)$ and $C_{\mathrm{it}}$ is given by $\frac{G_{P}(\omega)}{\omega}=\frac{C_{i t}}{2 \omega \tau_{i t}} \ln \left[1+\left(\omega \tau_{i t}\right)^{2}\right]$, where $\tau_{\mathrm{it}}$ is the relaxation time constant and $\tau_{i t}=C_{i t} R_{i t}$.

Hence, $C_{\text {it }}$ and $\tau_{\text {it }}$ can be deduced by the equations $C_{i t}=2.5\left(\frac{G_{P}}{\omega}\right)_{\max }$ and $\tau_{i t}=\frac{1.98}{2 \pi f_{0}}$, where $\left(\frac{G_{P}}{\omega}\right.$ )$_{\max }$ is the maximum value of $\frac{G_{P}}{\omega}$, and $f_{0}$ is the corresponding frequency at which the maximum is obtained (Figure S3).

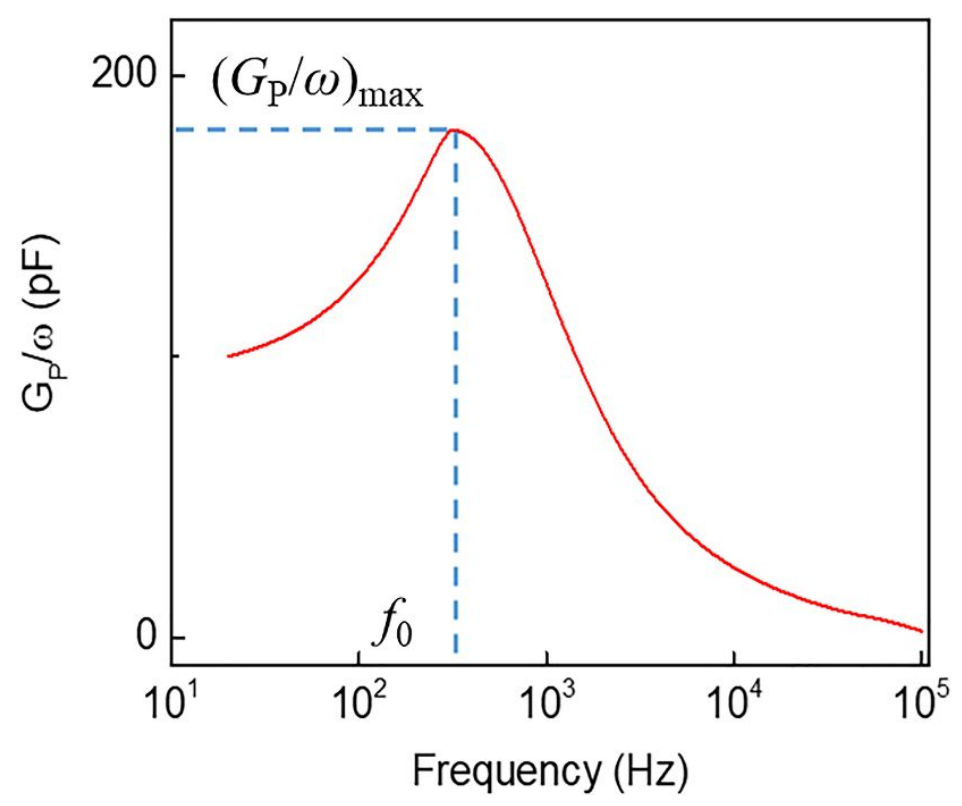

Figure S3. Schematic illustration of calculated $G_{\mathrm{P}} / \omega$ capacitance as a function of frequency for different gate biases. $\left(\frac{G_{P}}{\omega}\right)_{\max }$ and the corresponding frequency $f_{0}$ are shown. 


\section{Note 2. The radius of the polarons on $\mathrm{C}_{8}-\mathrm{BTBT} / \mathrm{HfO}_{2}$ and $\mathrm{C}_{8}-\mathrm{BTBT} / \mathrm{SiO}_{2}$ interface.}

The charge carriers in molecular crystalline semiconductors interact with the ionic polarizations of the dielectrics $\left(\mathrm{SiO}_{2}, \mathrm{HfO}_{2}\right.$, etc.), leading to the formation of Fröhlich polarons. ${ }^{2}$ The radius of the small Fröhlich polarons can be determined by the electron-phonon coupling, which is given by. ${ }^{3}$

$$
\lambda=\frac{E_{p}}{D}
$$

where $D$ is the half bandwidth, and the bandwidth $W_{a}^{h}(2 D)$ is $0.84 \mathrm{eV}$ along $a$ direction for holes. ${ }^{4}$ The value is larger than rubrene, ${ }^{5}$ DATT and DATT. ${ }^{4}$ The energy $E_{\mathrm{p}}$ can be written by a simplified expression as: ${ }^{2}$

$$
E_{p}=\frac{a_{B}}{z} \beta(R y d)
$$

where $z$ is the distance between the surface polaron and the dielectric, which is $0.8 \mathrm{~nm}$ for $\mathrm{C}_{8}$-BTBT semiconductors. $\beta=\left(\varepsilon_{s}-\varepsilon_{\infty}\right) /\left(\left(k+\varepsilon_{s}\right)\left(k+\varepsilon_{\infty}\right)\right)$ can quantified the ionic polarizability of the interface, expressed in terms of the measured dielectric constants of the gate dielectric $\left(\varepsilon_{\mathrm{s}}, \varepsilon_{\infty}\right)$ and of $\mathrm{C}_{8}$-BTBT $(\kappa)$. The values of $\beta$ for $\mathrm{SiO}_{2}$ and $\mathrm{HfO}_{2}$ are 0.051 and 0.097 , respectively ${ }^{2} \cdot a_{B}=0.53 \AA$ is the Bohr radius. Ryd is the Rydberg constant.

Based on above equations, $\lambda=0.109$ and 0.24 for $\mathrm{SiO}_{2}$ - and $\mathrm{HfO}_{2}$-based devices. Polaron radius $R_{\mathrm{p}}$ at different values of the electron-phonon coupling are summarized in Table S2. ${ }^{3}$ Hence, $R_{\mathrm{p}}$ is about $6 a(6 a \approx 4.2 \mathrm{~nm})$ for $\mathrm{SiO}_{2}$ and $3.7 a(3.7 a \approx 2.6 \mathrm{~nm})$. In practical devices, polaron radius will be a little smaller than the theoretical value, because a further effective reduction of the bandwidth can arise from thermal fluctuations. ${ }^{6}$ The polaron radius can be regarded as the inherent limit of the effective accumulation thickness (Figure S4). The results of the polaron radius are well consistent with the minimized $X_{\mathrm{ac}}$ for $\mathrm{HfO}_{2}(2.3 \mathrm{~nm})$ and $\mathrm{SiO}_{2}(4.4 \mathrm{~nm})$ at high $V_{\mathrm{G}}$ by the capacitance method, indicating the intrinsic correlation between the length of carrier distribution and the radius scale of the polarons.

Table S2. Polaron radius in units of the lattice spacing a for different values of the dimensionless electron-phonon coupling $\lambda$.

\begin{tabular}{cccccccccc}
\hline$\lambda$ & 0.1 & 0.2 & 0.4 & 0.7 & 1.0 & 1.2 & 1.4 & 1.6 & 2 \\
\hline$R_{\mathrm{p}} / a$ & 6.0 & 3.7 & 2.3 & 1.6 & 1.2 & 0.9 & 0.5 & 0.4 & 0.3 \\
\hline
\end{tabular}




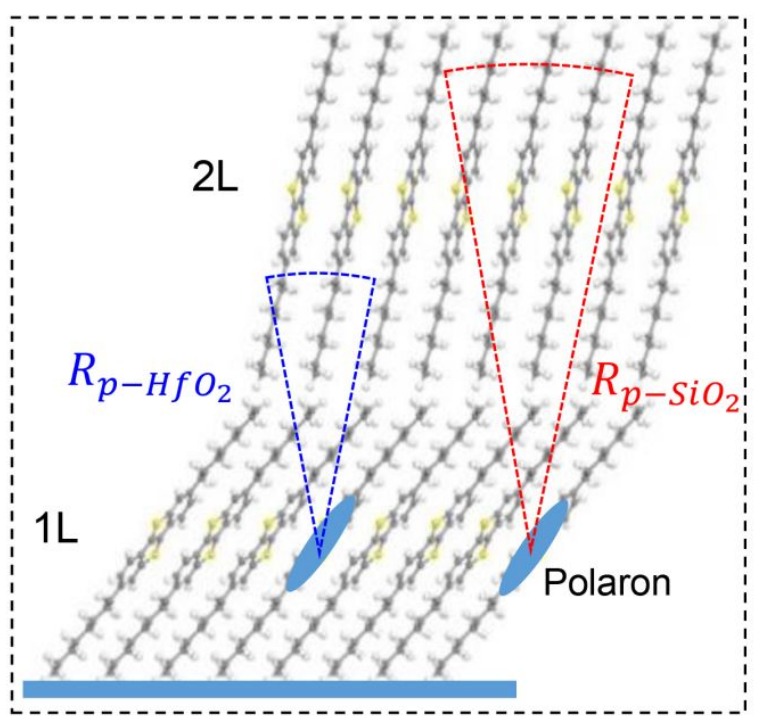

Figure S4. The radius of the polarons on $\mathrm{C}_{8}-\mathrm{BTBT} / \mathrm{HfO}_{2}$ and $\mathrm{C}_{8}-\mathrm{BTBT} / \mathrm{SiO}_{2}$ interface. The estimated polaron radius that formed at $\mathrm{C}_{8}-\mathrm{BTBT} / \mathrm{SiO}_{2}$ interface is $4.2 \mathrm{~nm}$, and changes to $2.6 \mathrm{~nm}$ at $\mathrm{C}_{8^{-}}$ $\mathrm{BTBT} / \mathrm{HfO}_{2}$ interface. The polaron radius can be regarded as the inherent limit of the effective accumulation thickness, hence the minimized thickness of carrier accumulation on $\mathrm{HfO}_{2}$ is the first layer (side-chain of $\mathrm{C}_{8}$-BTBT is insulator), and the distribution of carriers can extend to bilayer on $\mathrm{SiO}_{2}$. 


\section{Note 3. The density of states of the interface states at the band edge.}

The density of states can be described by $D_{i t}(E)=\alpha D_{0} \mathrm{e}^{\frac{E-E_{D}}{\varphi}}+D_{i t 0}$, where $D_{0}$ is the intrinsic density of states for perfect monolayer crystalline $\mathrm{C}_{8}$-BTBT, taken to $3.64 \times 10^{14} \mathrm{eV}^{-1} \mathrm{~cm}^{-2}$ and $3.72 \times 10^{14} \mathrm{eV}^{-1} \mathrm{~cm}^{-2}$ for $\mathrm{SiO}_{2}$ and $\mathrm{HfO}_{2}$ dielectric, respectively. It is roughly calculated by supposing the number of states in one of these bands is of the order of one state per molecule, so the density of states at the maximum is the molecular density of $\mathrm{C}_{8}$-BTBT divided by the width of the band $(\sim 1 \mathrm{eV})$. The lattice constant $a$ and $b$ of monolayer $\mathrm{C}_{8}$-BTBT on $\mathrm{SiO}_{2}$ is about 6.60 and $8.30 \AA$, the lattice constant $a$ and $b$ of monolayer $\mathrm{C}_{8}$-BTBT on $\mathrm{HfO}_{2}$ is about 6.57 and 8.28 $\AA$. A unit cell contains two molecules. So, the density of states per area is $3.6 \times 10^{14} \mathrm{eV}^{-1} \mathrm{~cm}^{-2}$ and $3.7 \times 10^{14} \mathrm{eV}^{-1} \mathrm{~cm}^{-2}$ for monolayer $\mathrm{C}_{8}$-BTBT crystal on $\mathrm{SiO}_{2}$ and $\mathrm{HfO}_{2}$ dielectric, respectively. $E_{\mathrm{D}}$ is the $\mathrm{HOMO}$ band edge. $\alpha$ is a fitted parameter $\left(2.75 \times 10^{-5}\right.$ for $\mathrm{SiO}_{2}$ and $3.5 \times 10^{-4}$ for $\left.\mathrm{HfO}_{2}\right) . \sigma$ is the energy width of the band tail states with the value of $65 \mathrm{meV}$ for $\mathrm{HfO}_{2}$ and $95 \mathrm{meV}$ for $\mathrm{SiO}_{2}$. 


\section{Note 4. The feasibility of Vissenberg-Matter model for carrier transport in $\mathrm{C}_{8}$-BTBT crystalline}

\section{films on the $\mathrm{HfO}_{2}$ substrate.}

The theory of Vissenberg-Matter model for the field-effect mobility in amorphous organic transistors is based on the variable range hopping (VRH) between localized states. The mechanisms limiting the carrier mobility in organic semiconductors involve the intrinsic quantum molecular overlapping and extrinsic factors including disorders, chemical impurities, and substrate polarizations. ${ }^{2,7-9}$ Firstly, for $\mathrm{C}_{8}$-BTBT crystalline films on a $\mathrm{HfO}_{2}$ substrate, the reduced height of the monolayer (1L) compared to bilayer (2L) suggests more tilted molecular packing. Recent studies revealed that the more tilted molecular packing in $1 \mathrm{~L}$ substantially modifies the spatial distribution of the bonding states, leading to weaker molecular $\pi$ - $\pi$ overlapping. ${ }^{10,11}$ Since carriers mainly transport within $1 \mathrm{~L}$ of $\mathrm{C}_{8}$-BTBT crystalline films on the $\mathrm{HfO}_{2}$ substrate, carriers are more localized in 1L with the mobility of $\sim 0.2 \mathrm{~cm}^{2} \mathrm{~V}^{-1} \mathrm{~s}^{-1}$, much smaller than the mobility of $\mathrm{C}_{8}$-BTBT single-crystals (up to 10 $\left.\mathrm{cm}^{2} \mathrm{~V}^{-1} \mathrm{~s}^{-1}\right) .{ }^{11,12}$ Secondly, although the deposited $\mathrm{C}_{8}$-BTBT crystalline films exhibited large-area morphology uniformity, there are still structure disorders and defects in the highly crystalline films, which hampering the carrier motions. ${ }^{13}$ Besides, the dynamic disorders, which possess a finite charge delocalization, also play a critical role in the charge transport properties of high-mobility $\mathrm{C}_{8}$-BTBT semiconductors. ${ }^{13,14}$ Hence, the extrinsic disorders can decrease the carrier mobility. Finally, the presence of a polarizable substrate of $\mathrm{HfO}_{2}$ can strongly affect the transport properties in FET. The carriers couple electrostatically to the polarization of the substrate, which provides an additional source of electron-phonon coupling, leading to the possible formation of polarons. ${ }^{2}$ The strong electronphonon coupling on the $\mathrm{HfO}_{2}$ substrate results in a thermally activated behavior. Taking these factors into consideration, we conclude that the charge transport on the $\mathrm{HfO}_{2}$ substrate is dominated by hopping with a low carrier mobility and a thermally activated behavior. Therefore, it is reasonable to utilize the Vissenberg-Matter model to describe and fitting the electrical transport of $\mathrm{C}_{8}$-BTBT crystalline films on the $\mathrm{HfO}_{2}$ substrate. Recently, Zhang et al validated that the $1 \mathrm{~L}$ devices exhibited insulating behavior and featured 2D hopping transport in highly ordered pentacene crystals using the Vissenberg-Matter model, which further verified the feasibility of the Vissenberg-Matter model for carrier transport in $\mathrm{C}_{8}$-BTBT crystalline films on the $\mathrm{HfO}_{2}$ substrate. ${ }^{10}$ 


\section{Note 5. Extracting 2D transport.}

Under a transistor structure, charge accumulation forms the conducting region as spatially within the first few molecular layers near the dielectric interface. The carrier accumulation behavior has a direct relationship with the charge transport properties. In the Vissenberg-Matters model, the conductivity is given by $\sigma(p)=A p^{\frac{T_{0}}{T}}$, where $p$ is the carrier density, $T_{0}$ is the width of the exponential distribution of the localized tail states, and $A=\sigma_{0}\left(\frac{\left(\frac{T_{0}}{T}\right)^{4} \sin \left(\pi_{\frac{T}{T_{0}}}\right)^{\frac{T_{0}}{T}}}{(2 \alpha)^{3} B_{c}}\right)$. In this equation, $\alpha^{-1}$ is the overlap localization length of the wave function, $\sigma_{0}$ is the conductivity prefactor, and $B_{c}$ is the critical number for the onset of percolation ( $B_{c}=2.8$ for $3 \mathrm{D}$ system and 4.48 for $2 \mathrm{D}$ systems).$^{15}$

For a 2D transport system, the carrier density is uniform as a function of distance from dielectric interface. Thus, the source-drain current is given as:

$$
I_{D S}^{2 D}=A \frac{W}{L}\left(d_{S C}\right)^{1-T_{0} / T}\left(\frac{C_{i}}{e}\right)^{T_{0} / T} \frac{T}{T_{0}+T}\left[\left(V_{T}-V_{G}\right)^{\left(T_{0} / T\right)+1}-\left(V_{T}-V_{G}+V_{D S}\right)^{\left(T_{0} / T\right)+1}\right]
$$

where $d_{\mathrm{sc}}$ is the semiconductor thickness, $W$ and $L$ are the width and length of the conducting channel, respectively, $V_{\mathrm{T}}$ is the threshold voltage, and $C_{\mathrm{i}}$ is the gate capacitance per unit area. The expression of current at high gate voltage bias reduces to a simplified power law by Taylor expansion, $I_{D S}^{2 D} \propto$ $\left(V_{T}-V_{G}\right)^{\frac{T_{0}}{T}}$. For each temperature, the power exponents can be obtained from $I_{D S^{-}} V_{G}$ characteristics and plotted as a function of $1 / T$. We can confirm the $2 \mathrm{D}$ transport model when the line crosses the exponent axis at the value of 0 .

The $I_{D S}$ for 3D transport can be given by $I_{D S}^{3 D} \propto\left(V_{T}-V_{G}\right)^{\frac{2 T_{0}}{T}}-1$. By fitting the power exponent as a function of $1 / T$, we can prove that $3 \mathrm{D}$ transport model applies when the intercept is -1 . 


\section{Note 6. The effect of traps on the charge carrier transport.}

Transistors with the ultrathin $\mathrm{C}_{8}$-BTBT crystalline films deposited by various methods exhibited different temperature-dependent transport behaviors, such as hopping transport, ${ }^{16}$ temperatureindependent transport, ${ }^{13}$ and bandlike transport (only down to $150 \mathrm{~K} \sim 200 \mathrm{~K}$ and replaced by the hopping transport at low temperatures). ${ }^{11}$ These observations are often related to molecular packing, disorders, and presence of traps due to structural defects and/or impurities, which influence the charge carrier mobilities. ${ }^{7}$

Even in high-quality $\mathrm{C}_{8}$-BTBT crystalline films, the structural defects can also be observed, which scatter the charge transport. ${ }^{13}$ We thus quantitatively estimated the density of traps of $\mathrm{C}_{8}$-BTBT films deposited by a floating-coffee-ring-driven assembly (this work) and a brush-coating method (our recent work $)^{13}$ in the same transistor structure and dielectric. The maximum density of interfacial traps ( $N_{\text {trap }}$ ) can be calculated from the values of the subthreshold swing $(S S), N_{\text {trap }}$ is given by $N_{\text {trap }}=C_{\mathrm{i}}$ $(q S S \log (e) / k T-1) / q$, where $q$ is the electronic charge, $S S$ is the subthreshold swing, $e$ is Euler's number, $k$ is the Boltzmann's constant, $T$ is the absolute temperature, and $C_{\mathrm{i}}$ is the gate dielectric capacitance. ${ }^{16}$ According to the transfer characteristics of transistors with bilayer (2L) $\mathrm{C}_{8}$-BTBT crystalline films on the $\mathrm{SiO}_{2}$ dielectric, the maximum trap density in our devices was $\sim 2.90 \times 10^{12} \mathrm{eV}^{-1} \mathrm{~cm}^{-2}$ (Figure S5a). This value was slightly higher than that obtained by a brush-coating method $\left(\sim 2.41 \times 10^{12} \mathrm{eV}^{-1} \mathrm{~cm}^{-2}\right) .{ }^{13}$ Considering the different transport behavior of these two transistors (hopping transport in films by floating-coffee-ring-driven assembly and temperature-independent transport in films by brush-coating method), a small change in trap densities was not enough to cause a transition in transport mode. Further studies revealed that a tuned molecular packing, especially molecular rotation, changes isotropic transfer integrals, leading to the different transport modes. ${ }^{13}$ Therefore, we believe that traps and molecular packing together lead to hopping transport in $\mathrm{C}_{8}$-BTBT crystalline films on the $\mathrm{SiO}_{2}$ interface.

The traps will not affect the conclusion of our manuscript, which focus on the change of carrier distributions and transport on different polar dielectrics. The estimated maximum trap densities of bilayer (2L) $\mathrm{C}_{8}$-BTBT crystalline films on $\mathrm{SiO}_{2}$ and $\mathrm{HfO}_{2}$ dielectrics were $\sim 2.90 \times 10^{12} \mathrm{eV}^{-1} \mathrm{~cm}^{-2}$ and $\sim 5.12 \times 10^{12} \mathrm{eV}^{-1} \mathrm{~cm}^{-2}$, respectively (Figure S5a,b). Hence, the values of the trap density on the two substrates were on the same order of magnitude. The trap states of both samples were further characterized by photoluminescence spectroscopy (Figure S5c). The extracted decay times $(\tau)$ for the $\mathrm{SiO}_{2}$-based device and $\mathrm{HfO}_{2}$-based one were $1.48 \pm 0.01$ and $1.36 \pm 0.01 \mathrm{~ns}$, respectively. ${ }^{13}$ The decay times suggested a higher trap-induced recombination rate in the $\mathrm{C}_{8}$-BTBT films of $\mathrm{HfO}_{2}$-based transistor, which was expected to possess a slightly higher trap density than the $\mathrm{SiO}_{2}$-based one. Considering significant increases in carrier mobility from a $\mathrm{SiO}_{2}$-based transistor to a $\mathrm{HfO}_{2}$-based one, the interfacial traps were not the intrinsic factor affecting the charge carrier distribution and transport 
on different dielectrics.

Table S3. Electrical performances, including $\mu_{\mathrm{FET}}$ (field-effect mobility), $S S$ (subthreshold swing), $V_{\mathrm{T}}$ (threshold voltage), and maximum trap density of the OFETs with different dielectrics.

\begin{tabular}{|c|c|c|c|c|}
\hline Dielectric & $\begin{array}{c}\mu_{\mathrm{FET}} \\
\left(\mathrm{cm}^{2} \mathrm{~V}^{-1} \mathrm{~s}^{-1}\right)\end{array}$ & $\left.S S(\mathrm{mV} \mathrm{dec})^{-1}\right)$ & $V_{\mathrm{T}}(\mathrm{V})$ & $\begin{array}{c}\text { Maximum Trap Density } \\
\left(\times 10^{12} \mathrm{eV}^{-1} \mathrm{~cm}^{-2}\right)\end{array}$ \\
\hline $\mathrm{SiO}_{2}$ & 6.10 & 480 & -7.5 & 2.90 \\
\hline $\mathrm{HfO}_{2}$ & 0.25 & 145 & -1.3 & 5.12 \\
\hline
\end{tabular}
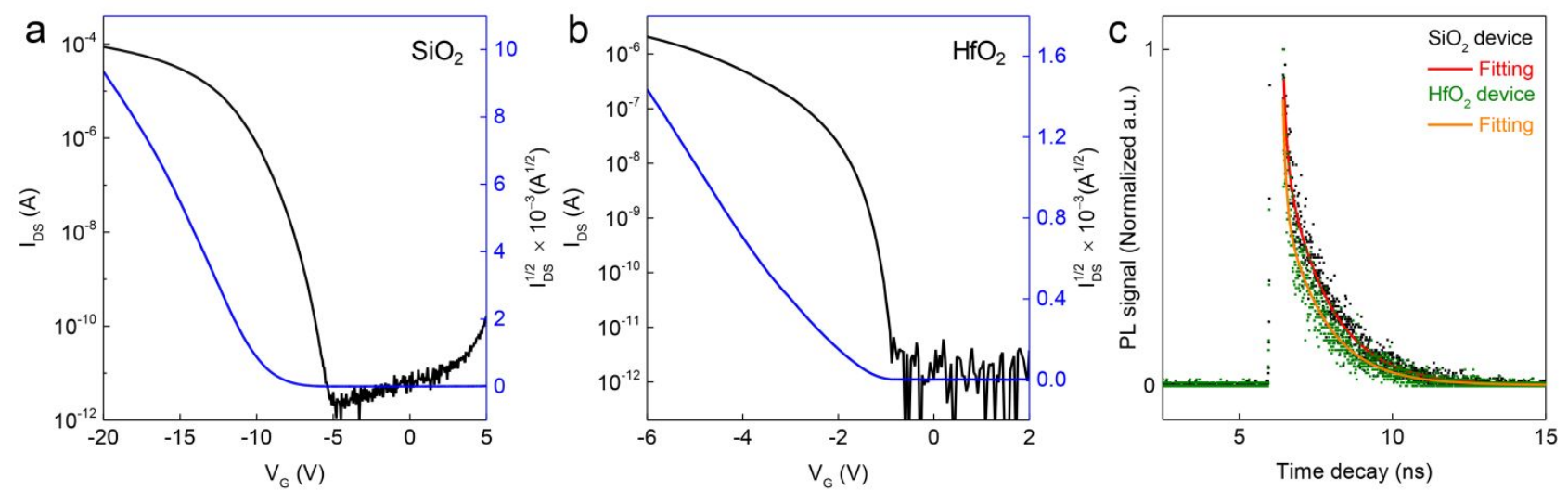

Figure S5. (a) Transfer characteristics of a $2 \mathrm{~L}$-based $\mathrm{C}_{8}$-BTBT OFET with a $\mathrm{SiO}_{2}$ dielectric at a drain voltage of $-20 \mathrm{~V}$. (b) Transfer characteristics of a 2L-based OFET with a $\mathrm{HfO}_{2}$ dielectric at a drain voltage of $-4 \mathrm{~V}$. (c) $\mathrm{PL}$ time decay for $\mathrm{SiO}_{2}$ and $\mathrm{HfO}_{2}$-based transistor. The time decays by the exponential fits of $\mathrm{SiO}_{2}$ and $\mathrm{HfO}_{2}$-based devices are $1.48 \pm 0.01 \mathrm{~ns}$ and $1.36 \pm 0.01 \mathrm{~ns}$, respectively. 


\section{References:}

(1) Nicollian, E. H.; Brews, J. R. MOS (Metal Oxide Semiconductor) Physics and Technology. Wiley, New York, 1982.

(2) Hulea, I. N.; Fratini, S.; Xie, H.; Mulder, C. L.; Iossad, N. N.; Rastelli, G.; Ciuchi, S.; Morpurgo, A. F. Tunable Fröhlich Polarons in Organic Single-Crystal Transistors. Nat. Mater. 2006, 5, 982-986.

(3) Fratini, S.; Xie, H.; Hulea, I. N.; Ciuchi, S.; Morpurgo, A. F. Current Saturation and Coulomb Interactions in Organic Single-Crystal Transistors. New J. Phys. 2008, 10, 033031.

(4) Xi, J.; Long, M.; Tang, L.; Wang, D.; Shuai, Z. First-Principles Prediction of Charge Mobility in Carbon and Organic Nanomaterials. Nanoscale 2012, 4, 4348.

(5) da Silva Filho, D. A.; Kim, E.-G.; Brédas, J.-L. Transport Properties in the Rubrene Crystal: Electronic Coupling and Vibrational Reorganization Energy. Adv. Mater. 2005, 17, 1072 1076.

(6) Troisi, A.; Orlandi, G. Charge-Transport Regime of Crystalline Organic Semiconductors: Diffusion Limited by Thermal Off-Diagonal Electronic Disorder. Phys. Rev. Lett. 2006, 96, 086601

(7) Coropceanu, V.; Cornil, J.; da Silva Filho, D. A.; Olivier, Y.; Silbey, R.; Brédas, J. L. Charge Transport in Organic Semiconductors. Chem. Rev. 2007, 107, 926-952.

(8) Heinz, B.; Anna, K. Charge Transport in Organic Semiconductors. Top. Curr. Chem. 2012, $312,1-66$.

(9) Wang, C.; Dong, H.; Jiang, L.; Hu, W. Organic Semiconductor Crystals. Chem. Soc. Rev. 2018, 47, 422-500.

(10) Zhang, Y.; Qiao, J.; Gao, S.; Hu, F.; He, D.; Wu, B.; Yang, Z.; Xu, B.; Li, Y.; Shi, Y.; Ji, W.; Wang, P.; Wang, X.; Xiao, M.; Xu, H.; Xu, J. Bin; Wang, X. Probing Carrier Transport and Structure-Property Relationship of Highly Ordered Organic Semiconductors at the TwoDimensional Limit. Phys. Rev. Lett. 2016, 116, 016602.

(11) He, D.; Qiao, J.; Zhang, L.; Wang, J.; Lan, T.; Qian, J.; Li, Y.; Shi, Y.; Chai, Y.; Lan, W.; Ono, L. K.; Qi, Y.; Xu, J. Bin; Ji, W.; Wang, X. Ultrahigh Mobility and Efficient Charge Injection in Monolayer Organic Thin-Film Transistors on Boron Nitride. Sci. Adv. 2017, 3, 1- 
9.

(12) Wang, Q.; Qian, J.; Li, Y.; Zhang, Y.; He, D.; Jiang, S.; Wang, Y.; Wang, X.; Pan, L.; Wang, J.; Wang, X.; Hu, Z.; Nan, H.; Ni, Z.; Zheng, Y.; Shi, Y. 2D Single-Crystalline Molecular Semiconductors with Precise Layer Definition Achieved by Floating-Coffee-Ring-Driven Assembly. Adv. Funct. Mater. 2016, 26, 3191-3198.

(13) Wang, Q.; Juarez-Perez, E. J.; Jiang, S.; Xiao, M.; Qian, J.; Shin, E.; Noh, Y.; Qi, Y.; Shi, Y.; Li, Y. Approaching Isotropic Transfer Integrals in Crystalline Organic Semiconductors. Phys. Rev. Mater. 2020, 4, 044604.

(14) Illig, S.; Eggeman, A. S.; Troisi, A.; Jiang, L.; Warwick, C.; Nikolka, M.; Schweicher, G.; Yeates, S. G.; Henri Geerts, Y.; Anthony, J. E.; Sirringhaus, H. Reducing Dynamic Disorder in Small-Molecule Organic Semiconductors by Suppressing Large-Amplitude Thermal Motions. Nat. Commun. 2016, 7, 10736.

(15) Brondijk, J. J.; Roelofs, W. S. C.; Mathijssen, S. G. J.; Shehu, A.; Cramer, T.; Biscarini, F.; Blom, P. W. M.; De Leeuw, D. M. Two-Dimensional Charge Transport in Disordered Organic Semiconductors. Phys. Rev. Lett. 2012, 109, 056601.

(16) Wang, Q.; Jiang, S.; Qian, J.; Song, L.; Zhang, L.; Zhang, Y.; Zhang, Y.; Wang, Y.; Wang, X.; Shi, Y.; Zheng, Y.; Li, Y. Low-Voltage, High-Performance Organic Field-Effect Transistors Based on 2D Crystalline Molecular Semiconductors. Sci. Rep. 2017, 7, 7830. 